\title{
Molecular Dynamics Simulation of Bamboo Heat Treatment with Cellulose Based on Molecular Different Weight Fractions of Water
}

\author{
Wei Wang, ${ }^{\mathrm{a}, *}$ Liyue Sun, ${ }^{\mathrm{a}}$ Mingshuai Wu, ${ }^{\mathrm{a}}$ Xing Li, ${ }^{\mathrm{a}}$ and Wenlong Song ${ }^{\mathrm{b}}$ \\ Eight groups of cellulose amorphous region models in which the mass \\ fraction of water was separately $0 \%, 1 \%, 2 \%, 3 \%, 4 \%, 5 \%, 6 \%$, and $7 \%$ \\ were established using a molecular dynamics software material studio. \\ The PCFF force field was selected to simulate the molecular dynamics of \\ the model under the constant-pressure and constant-temperature (NPT) \\ ensemble. The simulated temperature was set to $433.15 \mathrm{~K}$. The \\ experiment showed that the hydrogen bonds between cellulose chains \\ affected the structure of cellulose, which led to the change of the end-to- \\ end distance of the cellulose chain and the overall size of a cell. The \\ diffusion degree of water molecules was closely related to the number of \\ hydrogen bonds between cellulose and water. In the process of heat \\ treatment of bamboo, the present simulation results suggest that the \\ structure of bamboo may be damaged when the mass fraction of water \\ vapor reaches or exceeds $7 \%$.
}

Keywords: Molecular dynamics; Mechanical properties; Heat treatment; Mass fraction of water

Contact information: a: College of Engineering and Technology, Northeast Forestry University, Harbin, 150040, China; b: College of Mechanical and Electrical Engineering, Northeast Forestry University,

Harbin, 150040, China; *Corresponding author: cedar3@163.com

\section{INTRODUCTION}

Bamboo is an important renewable resource. Compared with wood, it has the characteristics of high strength, toughness, rigidity, and easy longitudinal division (Li et al. 2015). Bamboo heat treatment is a technique in which bamboo is placed in a heat transfer medium, such as air, inert gas, or water vapor, and subjected to short-term pyrolysis treatment at a high temperature of $160{ }^{\circ} \mathrm{C}$ to $250{ }^{\circ} \mathrm{C}$. The heat treatment of bamboo in water vapor plays an important role in improving the utilization and quality of bamboo because of the change of the internal structure of the bamboo material. The water absorption following treatment is decreased, and the dimensional stability increased, which can help to improve the stability of bamboo in a humid environment (Cao et al. 2012; Wang et al. 2018). The study found that the mass fraction of water vapor during heat treatment has a significant impact on the properties of bamboo after heat treatment.

Cellulose is the main component of bamboo that determines its structure and properties. Cellulose-based materials are also among the most important raw materials in industry. Researches have noted that every $1 \%$ increase in moisture content will double the rate of cellulose hydrolysis and aging in the operating environment of transformers (Lundgaard et al. 2004). Since the hydrolytic aging of cellulosic material is closely related to the moisture content and distribution, we consider that during the heat treatment of bamboo, the mass fraction of water vapor should not be too high, as that might be expected to reduce the mechanical properties of bamboo.

Wang et al. (2020). "Effect of water molecular weight," BioResources 15(3), 6766-6780. 6766 
Cellulose is a polymeric compound formed by polymerization of D-glucopyranose. Natural cellulose consists of crystalline and amorphous regions. The cellulose molecules in the crystalline zones are arranged in a compact and orderly manner. In the process of heat treatment with steam, the action of water molecules is limited to the surface of the crystallized region, and it cannot interact well with the crystalline zone (LeNeveu et al. 1976; Lee and Rossky 1994; Heiner et al. 1998). However, cellulose molecules in the amorphous region are irregularly distributed, and thus the adsorption of water molecules is strong. During heat treatment of cellulose in the presence of water, water molecules enter the cellulose amorphous region. Structural changes of cellulose occur, further affecting the mechanical properties of cellulose. Therefore, this paper selects the amorphous region as the object to study the variation of bamboo in the heat treatment process.

The repeating unit of natural cellulose is $\beta$-D-glucose. A cellulose chain passes through several crystal regions and amorphous regions. The length of the amorphous region in the cellulose chain is really small. When studying the properties of cellulose amorphous regions, Mazwau and Heux (2003) used cellulose chains with different degrees of polymerization (DP) to build an amorphous cellulose model. After a simulation experiment, it was found that there is no obvious difference in molecular conception or physical and chemical properties. Considering $\mathrm{DP}=40$ cellulose looks closer to nature, they chose $\mathrm{DP}=40$ cellulose chain to build an amorphous cellulose model. When studying the mechanical properties of amorphous cellulose, Chen et al. (2004) used a DP=20 cellulose chain to construct the amorphous region, and the obtained mechanical properties were basically consistent with the experimental values. Since the object of the present work is to study the effect of different moisture content on the mechanical properties of cellulose during the heat treatment of bamboo, $\mathrm{DP}=20$ was used as the length of the cellulose chain to find the suitable range of water vapor mass fraction during heat treatment of bamboo.

\section{EXPERIMENTAL}

\section{Methods}

Computational details

Molecular dynamics simulations were performed using Materials Studio software (Materials Studio, BIOVIA, v.8.0, San Diego, CA, USA). The amorphous region of cellulose was established using an amorphous polymer construction method (Theodorou and Suter 1985). Because the degree of polymerization has no remarkable effect on the physicochemical properties of the amorphous region of cellulose, a cellulose chain with a degree of polymerization of 20 was selected (Mazeau and Heux 2003). The change in cellulose in different mass fractions of water vapor was modeled using the Amorphous Cell (AC) Tools module. The PCFF force field was selected because it is suitable for the calculation of organic matter, like the cellulose chain and water vapor mixing system (Sun et al. 2018), the charge addition method uses the Ewald method; the Van der Waals addition method is an atom-based method. The target density was set to $1.5 \mathrm{~g} / \mathrm{cm}^{3}$ (Onyon 1972) to establish a water vapor-cellulose model.

The mass fraction of water vapor is $0 \%, 1 \%, 2 \%, 3 \%, 4 \%, 5 \%, 6 \%$, and $7 \%$, respectively. There was a cellulose molecular chain with a degree of polymerization of 20 in the model without water; 4 water vapor molecules were added and a cellulose molecular chain (DP $=20)$ in the model with $1 \%$ water; 8 water vapor molecules were added and a cellulose molecular chain ( $\mathrm{DP}=20)$ in the model with $2 \%$ water; 12 water vapor molecules 
were added and a cellulose molecular chain ( $\mathrm{DP}=20)$ in the model with $3 \%$ water; 16 water vapor molecules were added and a cellulose molecular chain $(\mathrm{DP}=20)$ in the model with $4 \%$ water; 20 water vapor molecules were added and a cellulose molecular chain (DP $=20$ ) in the model with 5\% water; 24 water vapor molecules were added and a cellulose molecular chain $(\mathrm{DP}=20)$ in the model with $6 \%$ water; 29 water vapor molecules and a cellulose molecular chain ( $\mathrm{DP}=20)$ were added to the model with $7 \%$ water. The simulated unit cell size was $19.6 \times 19.6 \times 19.6(\mathrm{~A} 3)$.

In Fig. 1, pink shows a cellulose molecular chain, and blue shows water molecules.

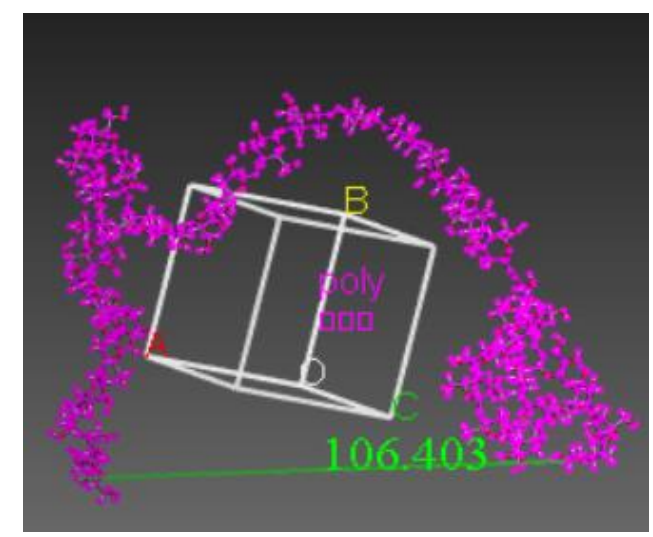

(a) Cellulose-water model without water

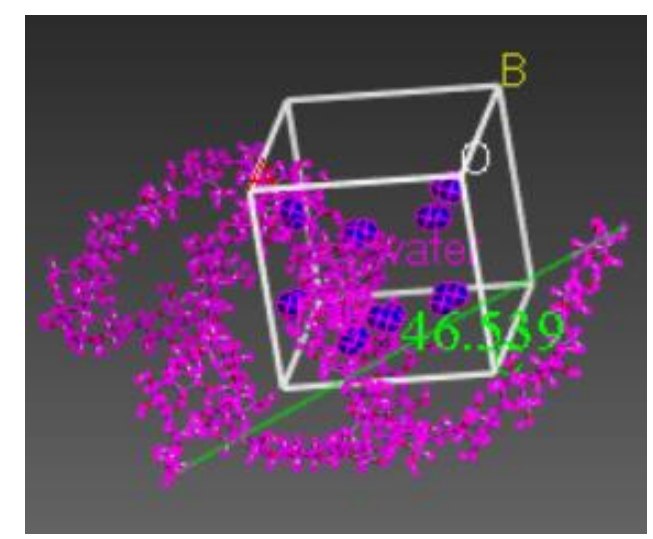

(c) Cellulose-water model with $2 \%$ water molecular

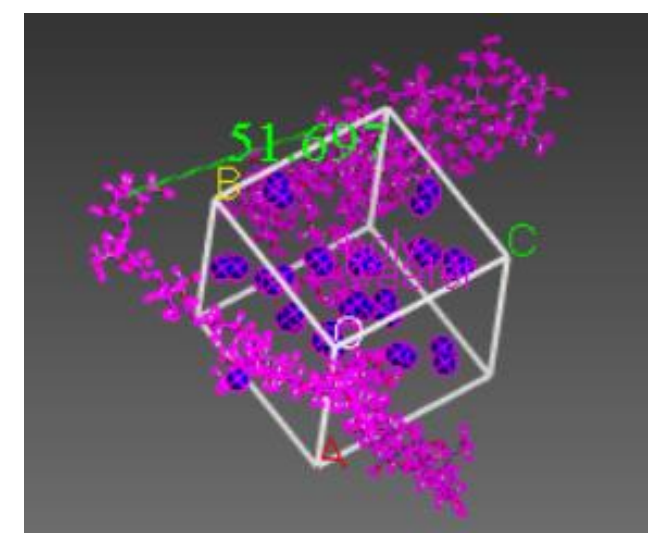

(e) Cellulose-water model with $4 \%$ water molecular

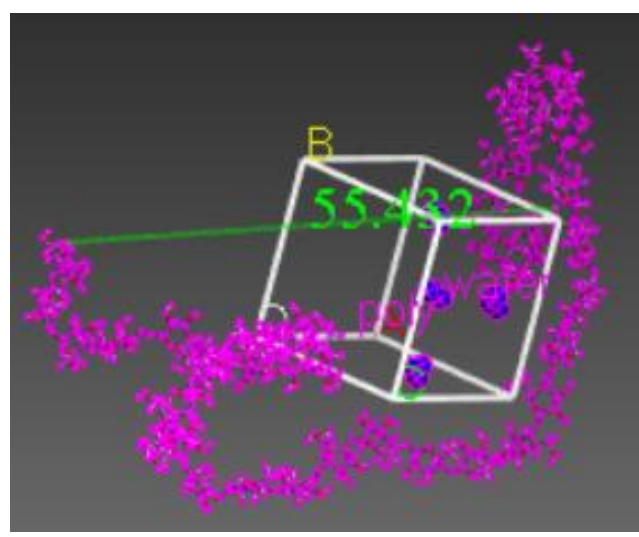

(b) Cellulose-water model with $1 \%$ water molecular

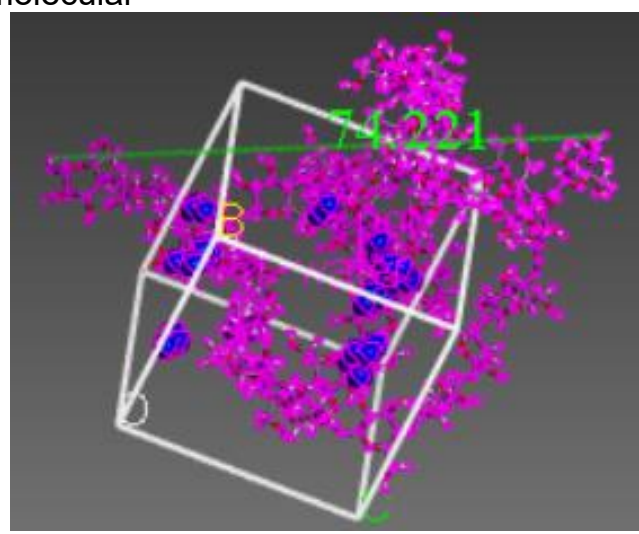

(d) Cellulose-water model with 3\% water molecular

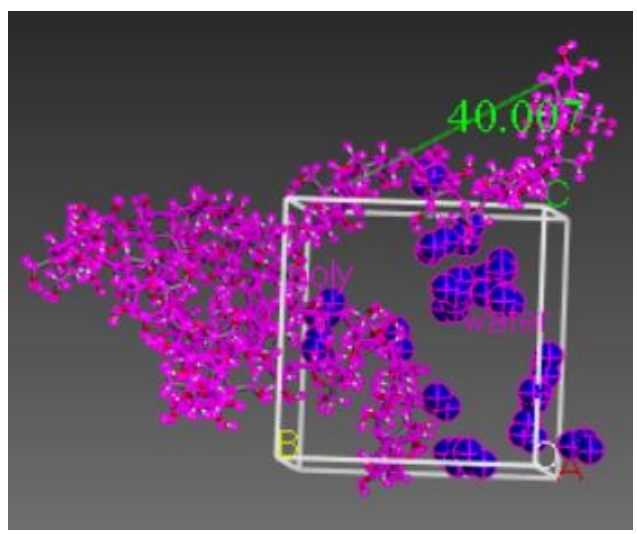

(f) Cellulose-water model with $5 \%$ water molecular 


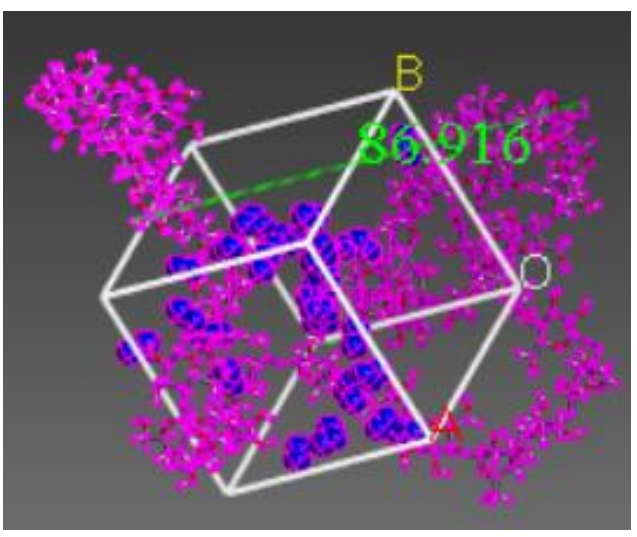

(g) Cellulose-water model with $6 \%$ water molecular

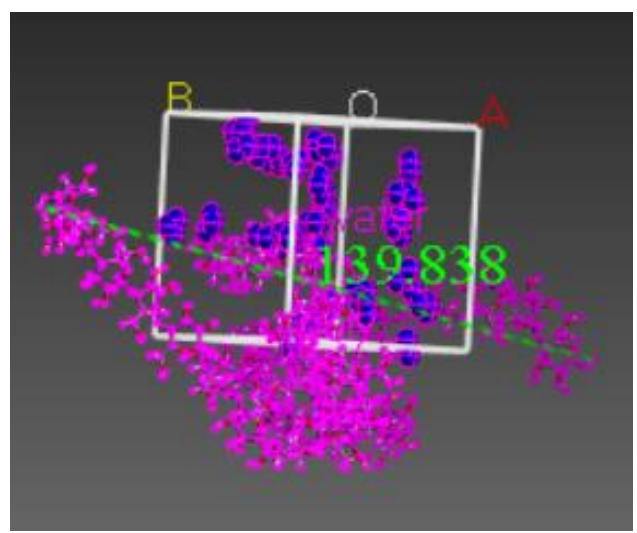

(h) Cellulose-water model with 7\% water molecular

Fig. 1. Modeled microstructures

\section{Dynamic simulation}

After the cellulose-water system model was obtained (shown in Fig. 1), the preliminary geometry optimization was performed using the calculation in the Forcite module, selecting the PCFF force field, and using the smart algorithm to calculate 1000000 steps, so the free motion of the mixed system molecules tend to balance and achieve energy minimization. Then, the model was simulated in $433.15 \mathrm{~K}$ in an isothermal-isobaric pressure ensemble (NPT). The initial velocity was set to random; the time step was $1 \mathrm{fs}$; the total length was 1000 ps, and the temperature control method was selected by Andersen (Zhang et al. 2013).

The Berendsen method (Iwona and Lea 2013) was used to control pressure; the electronic contribution was computed using the Ewald method (Ewald 1921), and the Van der Waals force was computed by atom-based calculations (Anderson 1980).

\section{RESULTS AND DISCUSSION}

\section{The balance of the system}

In the molecular dynamics (MD) simulation, temperature and energy were used as the basis of system equilibrium, as shown in Figs. 2 and 3 (the cellulose-water system model with $1 \%$ water as an example).

When the fluctuation range of the value was between $5 \%$ and $10 \%$, the system equilibrium was determined. The simulation accuracy can also be described by energy convergence parameters, which are defined as follows (Eq. 1),

$$
\delta E=\frac{1}{N} \sum_{i=1}^{N}\left|\frac{E_{i}-E_{0}}{E_{0}}\right|
$$

where the parameter $N$ refers to the total number of steps simulated. The parameter $E_{0}$ refers to the initial energy of the simulation system; the parameter $E_{\mathrm{i}}$ refers to the energy of the system after $i$ steps. When $\delta E \leq 0.001 \sim 0.003$, the simulation results are reliable. 


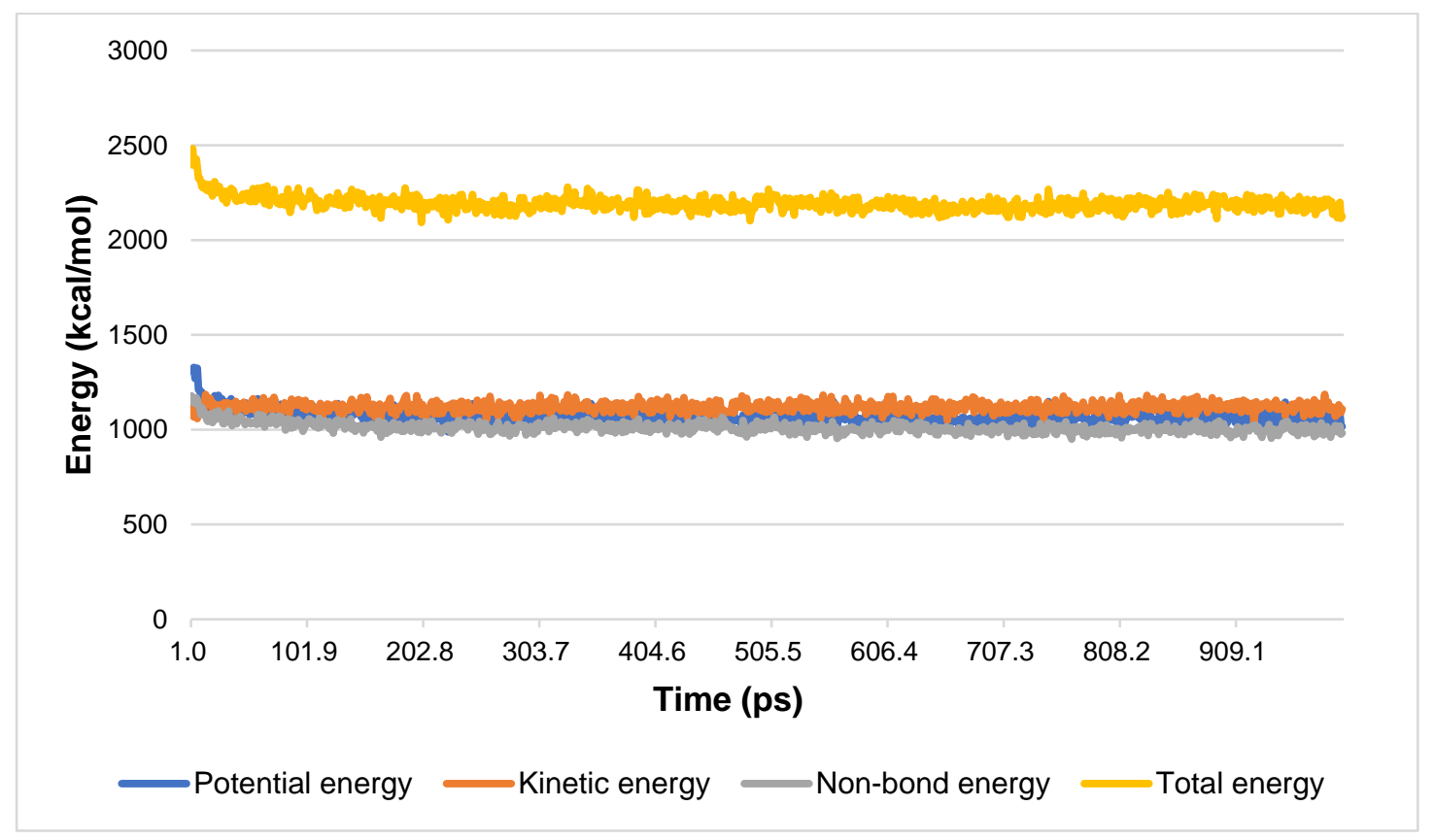

Fig. 2. Energy-time change graph

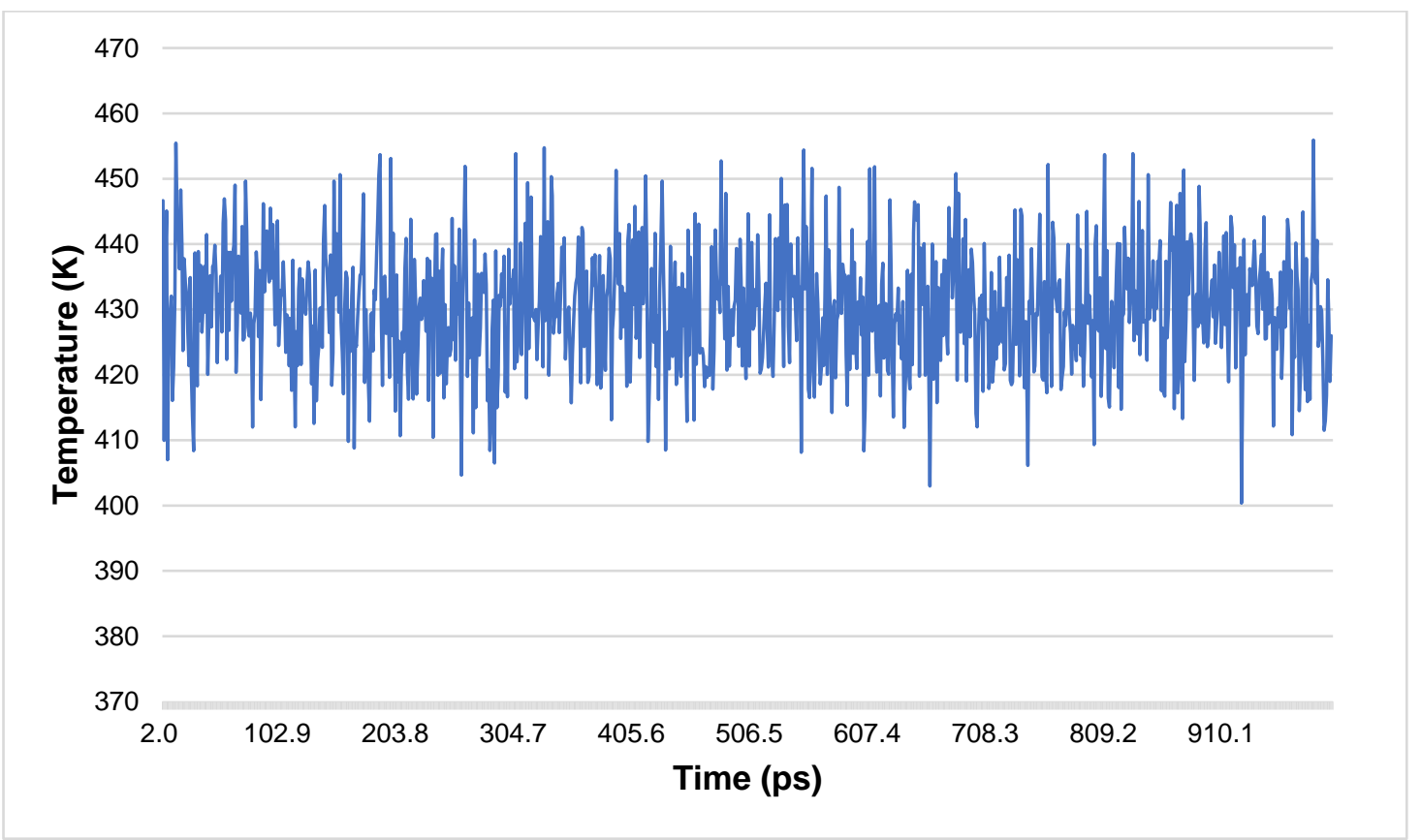

Fig. 3. Temperature-time change diagram

The MD simulation of the water molecule-cellulose mixed system with each mass fraction shows that the energy fluctuation of 100 ps was stable, and the fluctuating value and energy convergence parameters of the mixed system with each mass fraction of water molecule were as shown in Table 1. The energy fluctuation values of the last $100 \mathrm{ps}$ were between $0.6 \%$ to $4.2 \%$, the energy convergence parameters were between 0.0013 and 0.0020 . Therefore, after $1 \mathrm{~ns}$ molecular dynamics simulation, it can be considered that the system tends to be balanced and the simulation results are reliable. 
Table 1. Equilibrium Parameter Values of Cellulose-water System Under Different Mass Fractions

\begin{tabular}{|c|c|c|}
\hline Mass Fraction of Water & Fluctuation Value & Energy Convergence Parameter $(\delta E)$ \\
\hline $1 \%$ & $4.2 \%$ & 0.0015 \\
\hline $2 \%$ & $1.5 \%$ & 0.0013 \\
\hline $3 \%$ & $0.6 \%$ & 0.0013 \\
\hline $4 \%$ & $2.6 \%$ & 0.0025 \\
\hline $5 \%$ & $1.1 \%$ & 0.0017 \\
\hline $6 \%$ & $1.7 \%$ & 0.0014 \\
\hline $7 \%$ & $1.8 \%$ & 0.0020 \\
\hline
\end{tabular}

\section{Lattice parameters}

The cell formed by the cellulose-water system is a cube, and the cell parameters represent the length of the cell cube to describe the cell size. In this paper, the cellulosewater models all contained a cellulose chain $(\mathrm{DP}=20)$ and water molecules with mass fractions of $0 \%, 1 \%, 2 \%, 3 \%, 4 \%, 5 \%, 6 \%$, and $7 \%$.

After the molecular dynamic simulation, the cell parameters of cellulose-water, which are the length, width and height of the cell cube of cellulose-water molecules, are shown in the Table 2.

Table 2. Cell Parameters of Cellulose-water Model Under Different Mass Fraction

\begin{tabular}{|c|c|c|c|}
\hline \multirow{2}{*}{ Mass Fraction of Water } & \multicolumn{3}{|c|}{ Cell Parameters } \\
\cline { 2 - 4 } & The Length & The Width & The Height \\
\hline $0 \%$ & 20.10 & 20.10 & 20.10 \\
\hline $1 \%$ & 20.19 & 20.19 & 20.19 \\
\hline $2 \%$ & 20.20 & 20.20 & 20.20 \\
\hline $3 \%$ & 20.28 & 20.28 & 20.28 \\
\hline $4 \%$ & 20.45 & 20.45 & 20.45 \\
\hline $5 \%$ & 20.50 & 20.50 & 20.50 \\
\hline $6 \%$ & 20.57 & 20.57 & 20.57 \\
\hline $7 \%$ & 20.66 & 20.66 & 20.66 \\
\hline
\end{tabular}

From the data in the Table 2, the length of the lattice increased from 20.10 to 20.66 with the water molecules added. From Fig. 4, it can be clearly seen that with the increase of the mass fraction of water, the overall cell size of the cellulose-water model showed an upward trend (dotted line is the trendline), which proved that the water absorption of the cellulose chain was enhanced.

The number of hydrogen bonds

The properties of cellulose are mainly due to the high degree of polymerization of cellulose and the numerous hydrogen bonds among cellulose molecules (Hinterstoisser $e t$ al. 2003). The heat treatment process of the cellulose-water system can be analyzed by the Chain Flexibility theory (mainly hydrogen bond action). Adding water molecules to the cellulose chain structure will destroy the hydrogen bond between the original chains of cellulose and form the hydrogen bond between the cellulose chain and water. 


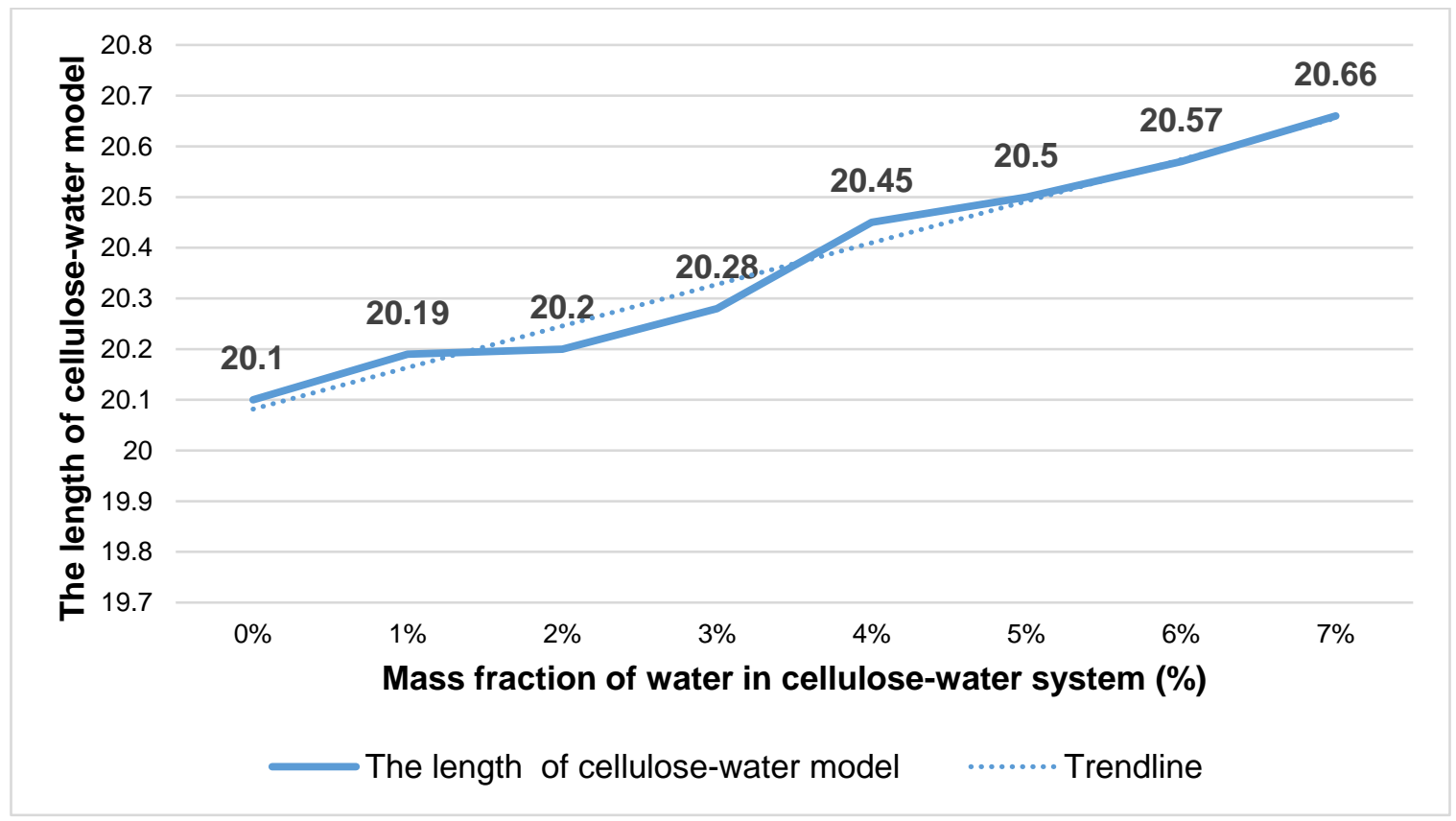

Fig. 4. Variation of cell size with the mass fraction of water in cellulose-water model

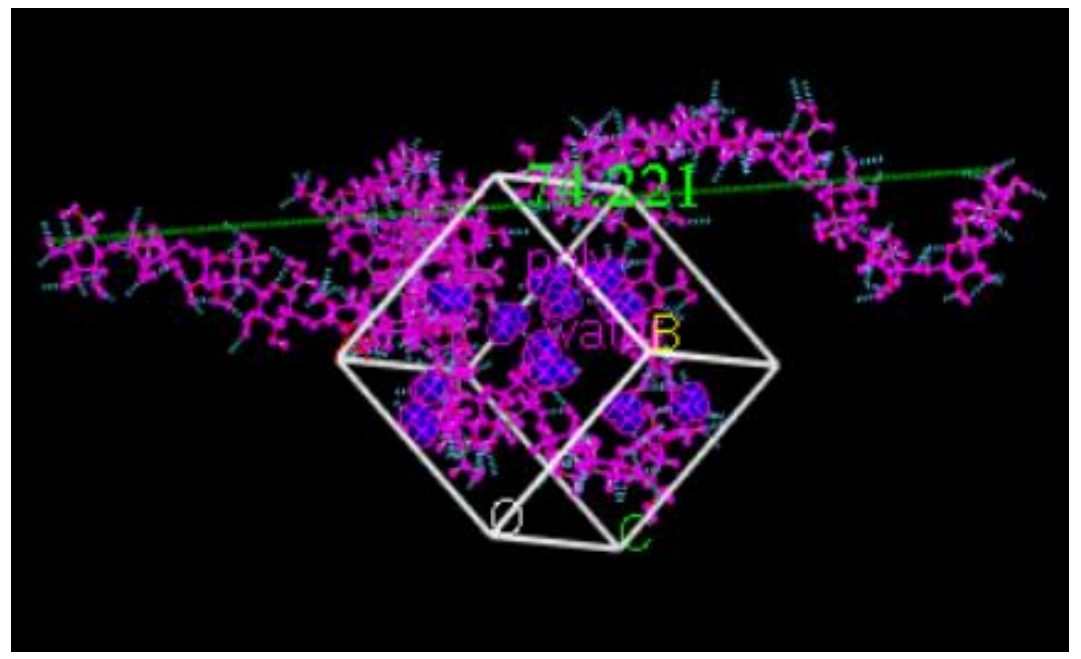

Fig. 5. The hydrogen bonds in the model

The hydrogen bonds in the cellulose-water system model were divided into intermolecular hydrogen bonds and intramolecular hydrogen bonds (shown as Fig. 5). Intramolecular hydrogen bonds refer to the hydrogen bonds formed in the cellulose chain, and the intermolecular hydrogen bonds refer to the hydrogen bonds formed between the cellulose chain and the water molecule. Intramolecular hydrogen bonds play an important role in the thermal stability and mechanical properties of cellulose molecules. Table 3 shows the number of hydrogen bonds in the cellulose-water system. It can be seen from Table 3 that the number of hydrogen bonds between cellulose molecules was between 100 and 142, and the number of hydrogen bonds between cellulose and water molecules did not exceed 12. Thus, there were more hydrogen bonds between cellulose molecules than 
intramolecular hydrogen bonds between the cellulose chain and water molecules in each cellulose-water system model with different water content, which fully indicated that the hydrogen bonds between cellulose chains played an important role in maintaining the stability of the cellulose structure and mechanical properties.

Figure 6 shows the trend diagram of all kinds of hydrogen bonds. The good performance of cellulose is mainly due to the high degree of polymerization of cellulose and the numerous hydrogen bonds in cellulose. The number of hydrogen bonds between cellulose chains increased at first from 130 to 142, showing that a small number of water molecules made more hydrogen bonds inside the cellulose molecules, causing the cellulose molecules to gain more excellent mechanical properties. And then the number of hydrogen bonds decreased to 120 , indicating that while too much water added they would destroy the structure of cellulose chains and caused aging degradation of bamboo. This also confirmed the change of the trend of the end-to-end distance of cellulose shortening and then increasing.

Table 3. Number of Hydrogen Bonds in the Cellulose-water System

\begin{tabular}{|c|c|c|c|c|}
\hline $\begin{array}{c}\text { Mass Fraction } \\
\text { of Water }\end{array}$ & $\begin{array}{c}\text { Total Number of } \\
\text { Hydrogen } \\
\text { Bonds }\end{array}$ & $\begin{array}{c}\text { Number of } \\
\text { Hydrogen } \\
\text { Bonds Between } \\
\text { Cellulose } \\
\text { Molecules }\end{array}$ & $\begin{array}{c}\text { Number of } \\
\text { Hydrogen } \\
\text { Bonds Between } \\
\text { Water } \\
\text { Molecules }\end{array}$ & $\begin{array}{c}\text { Number of } \\
\text { Hydrogen } \\
\text { Bonds Between } \\
\text { Cellulose and } \\
\text { Water } \\
\text { Molecules }\end{array}$ \\
\hline $0 \%$ & 130 & 130 & 0 & 0 \\
\hline $1 \%$ & 146 & 136 & 0 & 10 \\
\hline $2 \%$ & 155 & 130 & 0 & 25 \\
\hline $3 \%$ & 173 & 142 & 2 & 29 \\
\hline $4 \%$ & 173 & 115 & 3 & 55 \\
\hline $5 \%$ & 160 & 100 & 7 & 53 \\
\hline $6 \%$ & 193 & 120 & 11 & 62 \\
\hline $7 \%$ & 210 & 121 & 12 & 77 \\
\hline
\end{tabular}

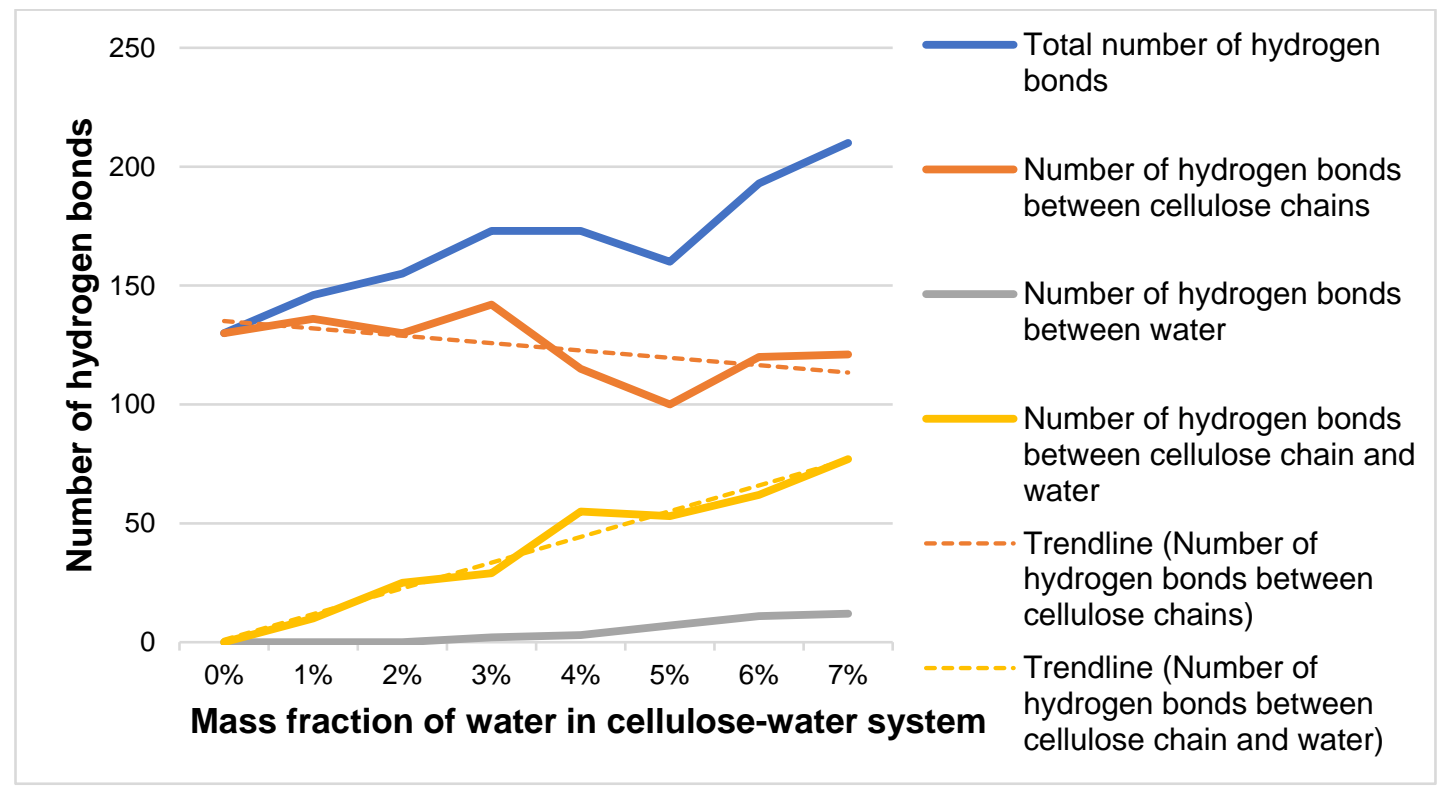

Fig. 6. The number of hydrogen bonds in cellulose-water system 
The mean square displacement and the molecular diffusion coefficient

The mean square displacement (MSD) is the mean squared sum of the position vector increments of a gas molecule after a simulation time in the system. The MSD can effectively characterize the migration path and behavior of molecules and plays a role in understanding the diffusion changes of gas molecules. It is represented by Eq. 2,

$$
\mathrm{MSD}=\sum_{i=1}^{n}\left\langle\left|\vec{r}_{i}(t)-\vec{r}_{i}(0)\right|^{2}\right\rangle
$$

where $<>$ indicates the average value, which indicates the centroid displacement of the $i$ th particle after a certain simulation time and the particle at the initial time, and $n$ is the number of diffusion particles. The molecular diffusion coefficient $D$ is often used to describe the diffusion behavior of small gas molecules. The expression is:

$$
D=\frac{1}{6 n} \lim _{t \rightarrow \infty} \frac{d}{d t} \sum_{i=1}^{n}\left\langle\left|r_{i}(t)-r_{i}(0)\right|^{2}\right\rangle
$$

It can be further simplified as Eq. 4,

$$
D=m / 6
$$

where $m$ is the slope obtained by fitting the MSD curve using the least squares method.

Figure 7 shows the MSD of water vapor molecules in a model with different mass fractions of water molecules. The MSD curve of water vapor molecules in different models in Fig. 6 was fitted to obtain the slope $m$ and the goodness of fit, and the gas small molecule diffusion coefficient $D$ of each model was calculated according to Eq. 4 as shown in Table 4.

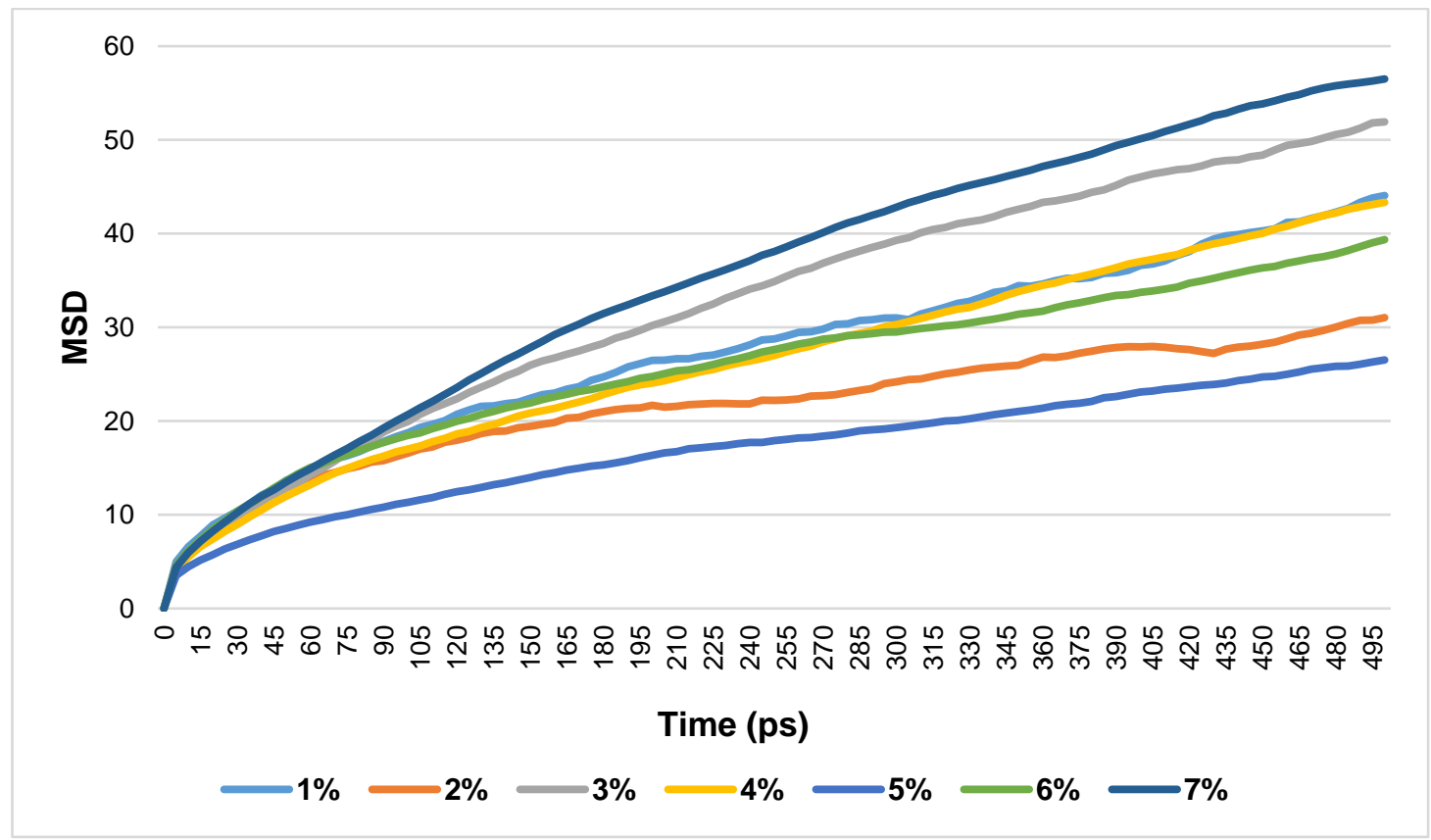

Fig. 7. Mean square displacement of water molecules 
Table 4. Diffusion Coefficient of Water Molecules in Cellulose-water System

\begin{tabular}{|c|c|c|c|c|c|c|c|c|}
\hline \multicolumn{2}{|c|}{\begin{tabular}{c} 
Mass Fraction of Water \\
\hline $\begin{array}{c}\text { Diffusion Coefficient } \\
\left(\mathrm{A}^{2} / \mathrm{ps}\right)\end{array}$
\end{tabular}} & 0.0104 & 0.0062 & 0.0140 & 0.0111 & 0.0062 & 0.0084 & 0.0158 \\
\hline & SSE & 12.93 & 15.2 & 21.74 & 7.722 & 4.77 & 16.58 & 753.3 \\
\cline { 2 - 9 } & R-square & 0.9987 & 0.9963 & 0.9988 & 0.99933 & 0.9987 & 0.9979 & 0.9666 \\
\cline { 2 - 9 } \\
$\begin{array}{c}\text { Goodness } \\
\text { of Fitting }\end{array}$ & $\begin{array}{c}\text { Adjust R- } \\
\text { square }\end{array}$ & 0.9987 & 09963 & 0.9988 & 0.9993 & 0.9987 & 0.9979 & 0.9963 \\
\cline { 2 - 9 } & RMSE & 0.3615 & 0.3919 & 0.4686 & 0.2793 & 0.2195 & 0.4093 & 2.759 \\
\hline
\end{tabular}

Where SSE is sum of squares due to error, and RMSE is root mean square error

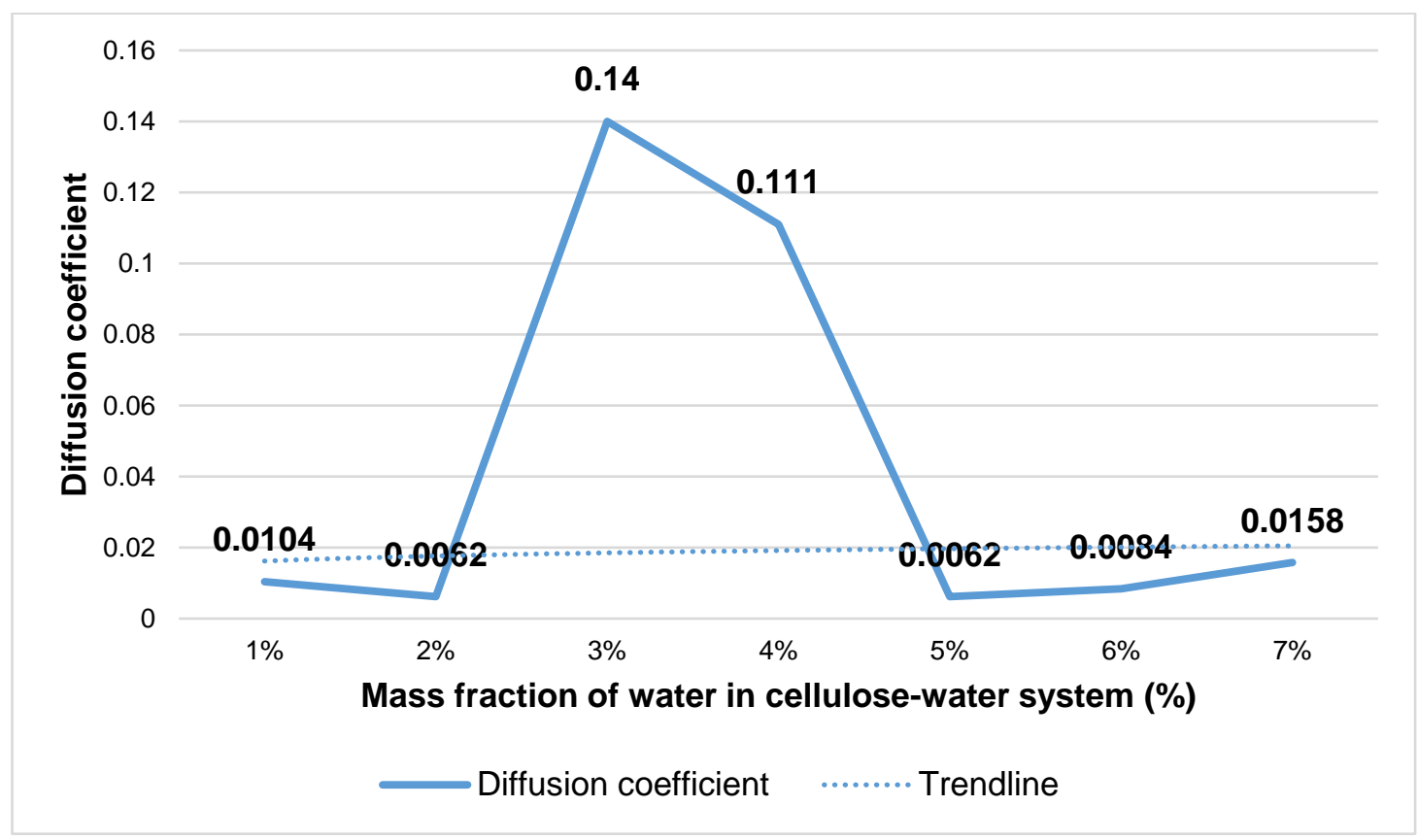

Fig. 8. Diffusion coefficient of water molecules in cellulose-water system

It can be seen from Fig. 8 and Table 4 that the overall trend of the diffusion coefficient of water molecules increased from 0.0104 to 0.0158 , but there was an abnormal value when the mass fraction of water was $3 \%$ and $4 \%$. The reason for this could have been that when the mass fraction of the water molecule was only $1 \%$ or $2 \%$, the Van der Waals force between the cellulose and water molecules could adsorb water molecules. However, when the mass fraction of the water molecules reached 3\%, the Van der Waals force between the molecules was not sufficient to attract the water molecules due to the increase of the water molecule content, and the number of hydrogen bonds was not noticeable, so the diffusion of water molecules was obviously enhanced. When the mass fraction of water molecules was as large as $5 \%$, the number of hydrogen bonds increased so that the hydrogen bonds played a role in attracting the water molecules again, so that the diffusion coefficient was again reduced to 0.0062 . 


\section{End-to-end distance}

The more flexible sugar linkages in the cellulose molecule determine the flexibility of the cellulosic material. The length between the chain end and the end of the cellulose molecule will vary from time-to-time due to the thermal motion of the molecule. The endto-end distance, which is the distance between the leading end and the end of the chain, describes the degree of aggregation and contraction of the chain. The end-to-end distance is an important parameter to measure the thermal motion of cellulose molecules. Table 5 shows the end-to-end distance range of cellulose molecules under different water molecular mass fractions. If the end-to-end distance of the cellulose molecular chain changes greatly, it means that the molecular structure inside the cellulose material changes seriously. Figure 9 shows the change trend of the end distance of the cellulose chain, which decreased at first from 109.4 to 48.7 and then increased from 47 to 144.2 , which was consistent with the change trend of the hydrogen bond number between cellulose chains. The abnormal value appeared when the mass fraction of water was $3 \%$. It is speculated that it may have been caused by the force between cellulose molecules and water molecules just like the change of the diffusion coefficient of water molecules.

Table 5. The End-to-end Distance in the Cellulose-water System

\begin{tabular}{|c|c|c|}
\hline Mass Fraction of Water & $\begin{array}{c}\text { Range of the End-to-end } \\
\text { Distance of Cellulose Chain }\end{array}$ & $\begin{array}{c}\text { End-to-end Distance } \\
\text { Weighted Average }\end{array}$ \\
\hline $0 \%$ & 106.40 to 111.27 & 109.42 \\
\hline $1 \%$ & 54.67 to 63.99 & 60.92 \\
\hline $2 \%$ & 44.96 to 50.22 & 48.67 \\
\hline $3 \%$ & 74.07 to 81.70 & 79.35 \\
\hline $4 \%$ & 51.70 to 62.60 & 60.05 \\
\hline $5 \%$ & 40.01 to 50.17 & 47.00 \\
\hline $6 \%$ & 86.07 to 91.59 & 89.76 \\
\hline $7 \%$ & 139.90 to 145.92 & 144.18 \\
\hline
\end{tabular}

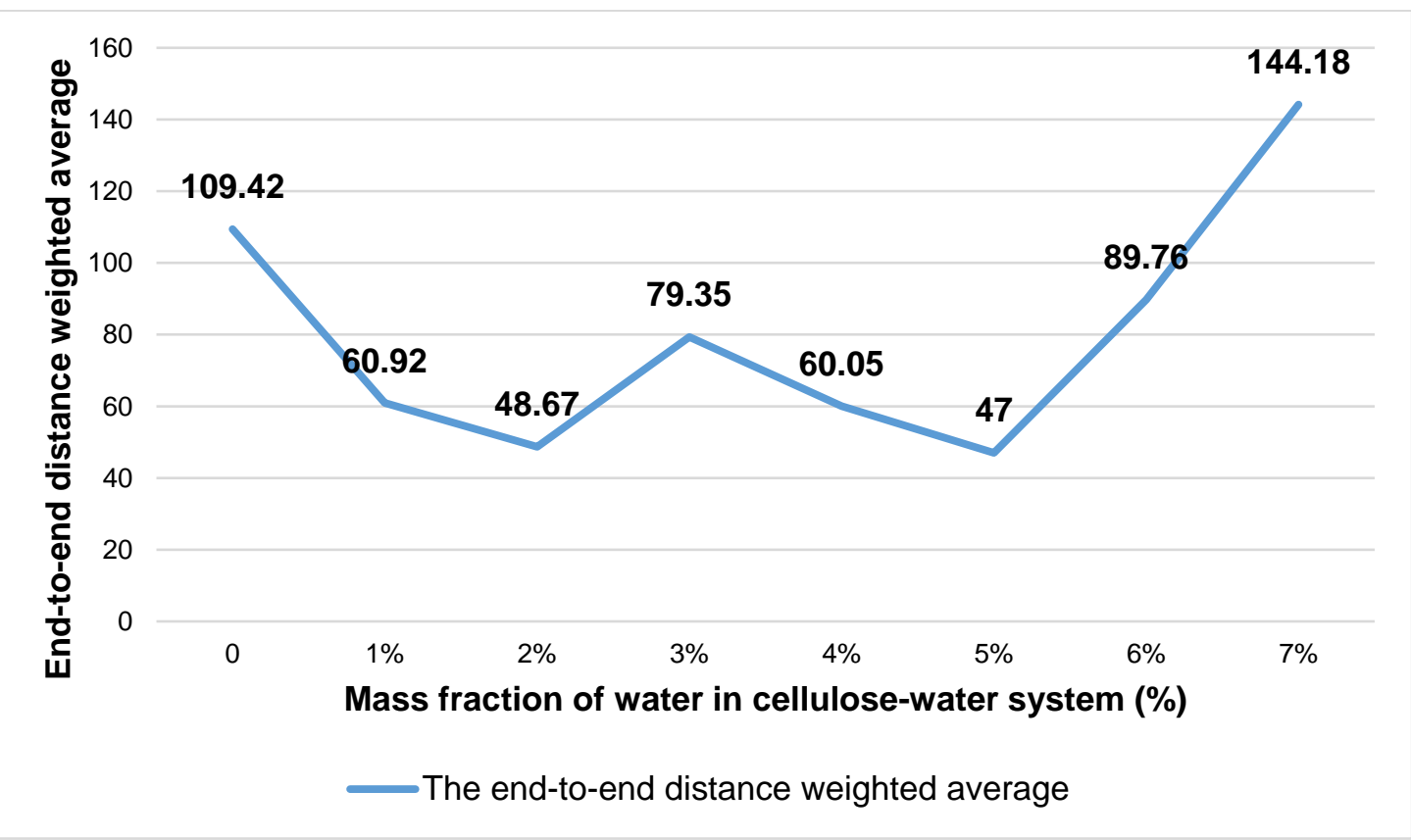

Fig. 9. The end-to-end distance of the cellulose chain 


\section{Mechanical property}

The kinetic simulation results at $160{ }^{\circ} \mathrm{C}$ were calculated and the mechanical parameters obtained were able to characterize the mechanical properties of the bamboo. Because the amorphous region of cellulose can be considered isotropic, its mechanical properties can be calculated by $C_{11}$ and $C_{12}$, so that Eq. 5,

$$
\lambda=C_{12}
$$

and Eq. 6,

$$
\mu=\left(C_{11}-C_{12}\right) / 2
$$

where $\lambda$ and $\mu$ are called Lame constants. The value of $C_{\mathrm{ij}}$ is given by Eq. 7:

$$
\left[C_{i j}\right]=\left[\begin{array}{cccccc}
\lambda+2 \mu & \lambda & \lambda & 0 & 0 & 0 \\
\lambda & \lambda+2 \mu & \lambda & 0 & 0 & 0 \\
\lambda & \lambda & \lambda+2 \mu & 0 & 0 & 0 \\
0 & 0 & 0 & \mu & 0 & 0 \\
0 & 0 & 0 & 0 & \mu & 0 \\
0 & 0 & 0 & 0 & 0 & \mu
\end{array}\right]
$$

The Lame constant is used to calculate mechanical properties such as Young's modulus $E$, shear modulus $G$, bulk modulus $K$, and Poisson's ratio $\gamma$. The formula is as follows (Eq. 8):

$$
E=\frac{\mu(3 \lambda+2 \mu)}{\mu+\lambda}, G=\mu, K=\lambda+\frac{2}{3 \mu}, \gamma=\frac{\lambda}{2(\lambda+\mu)}
$$

The mechanical properties of the cellulose-water system model under different mass fractions of water were obtained by MD calculation, as shown in Table 6. Young's modulus is the ratio of the longitudinal stress to strain of the material, that is, the longitudinal elastic modulus, which measures the rigidity of the material, and its performance is proportional to the numerical value. The shear modulus is the ratio of shear stress to strain; and the bulk modulus is a measure of the incompressibility of a solid material. Poisson's ratio is the ratio of transverse stress to strain, that is, the transverse elastic modulus, characterizing the plasticity of the material.

Table 6. Mechanical Properties of Cellulose-water System

\begin{tabular}{|c|c|c|c|c|c|c|}
\hline Mass Fraction of Water & $\lambda(\mathrm{Pa})$ & $\mu(\mathrm{Pa})$ & $E(\mathrm{~Pa})$ & $G(\mathrm{~Pa})$ & $K(\mathrm{~Pa})$ & $r$ \\
\hline $0 \%$ & 7.56 & 0.64 & 1.86 & 0.637 & 8.60 & 0.46 \\
\hline $1 \%$ & 6.67 & 4.78 & 12.35 & 4.78 & 6.81 & 0.29 \\
\hline $2 \%$ & 2.07 & 4.57 & 10.56 & 4.57 & 2.22 & 0.16 \\
\hline $3 \%$ & 77.78 & 4.35 & 12.81 & 4.35 & 77.93 & 0.47 \\
\hline $4 \%$ & 6.21 & 4.73 & 12.15 & 4.73 & 6.35 & 0.28 \\
\hline $5 \%$ & 11.69 & 3.17 & 8.84 & 3.17 & 11.90 & 0.39 \\
\hline $6 \%$ & 5.40 & 3.78 & 9.79 & 3.78 & 5.58 & 0.29 \\
\hline $7 \%$ & -13.81 & 3.89 & 13.17 & 3.89 & -13.64 & 0.70 \\
\hline
\end{tabular}

It can be seen from Table 6 and Fig. 10 that with the increase of the mass fraction of water, the Young modulus $(E)$, shear modulus $(G)$, and Poisson's ratio $(r)$ of a cellulose chain did not change obviously, but the volume modulus $(K)$ had a remarkable change. When the mass fraction of water was $3 \%$, it increased greatly at 77.78 , which led to the 
increase of the volume modulus due to the large number of hydrogen bonds between cellulose chains and the compact structure. When the mass fraction of water reached $7 \%$, the volume modulus became -13.81 (to be negative), which is impossible in practical applications. The structure of bamboo was seriously destroyed when the mass fraction of water reached $7 \%$. This conclusion was in good agreement with the end-distance of the cellulose chain and the diffusion coefficient of water molecules. It is also consistent with the conclusion that water will age and hydrolyze cellulose, which Lungfaard et al. (2004) studied earlier.

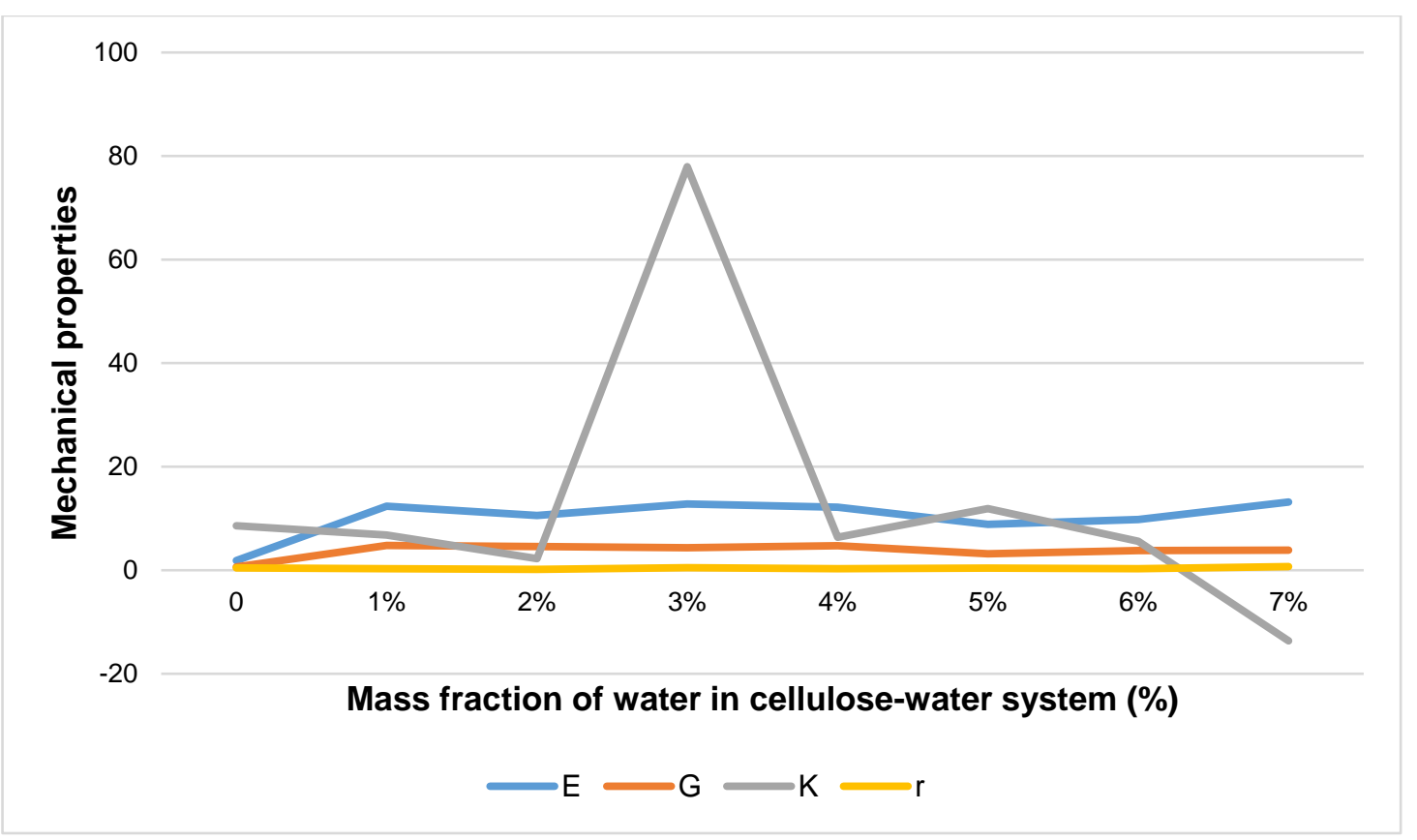

Fig. 10. The trendline of mechanical properties of cellulose-water system

Based on the above analysis and discussion, the authors suspected that when the cellulose is heat-treated in a water vapor environment, the mass fraction of water vapor should not be too high, since that might destroy the structure of bamboo. The analysis showed that the mass fraction of water vapor must not exceed $7 \%$. It is probably the best for the mechanical properties of bamboo when the mass fraction of water vapor is about $3 \%$.

Since the experimental samples usually have been raw bamboo, it was difficult to ignore the other chemical contents, such as hemicellulose and lignin, when studying the effects of the heat treatment on cellulose. The molecular simulation method can be used to construct the amorphous region model and the diffusion of small gas molecules at different conditions (Miyamoto et al. 2016). The present simulation provides theoretical support for the research of others in the future.

\section{CONCLUSIONS}

1. Models of cellulose-water were established through molecular dynamics simulations to explore the appropriate mass fraction range of water vapor in the amorphous region of cellulose, which is the main component of bamboo. 
2. The change trend of cell size and end-to-end distance of the cellulose chain was consistent with that of hydrogen bond number between cellulose chains. With the increase of the mass fraction of water, the number of hydrogen bonds between cellulose and cellulose chains increased at first and then decreased, and the overall cell size tended to rise, and the end-to-end distance of the cellulose chain first shortened and then increased. The hydrogen bond between cellulose chains affected the morphology of cellulose. The change of structure led to the change of the end-to-end distance of the cellulose chain and the size of cell.

3. The diffusion coefficient of water molecules was approximately the same as the change trend of hydrogen bond number between cellulose and water. The diffusion degree of water molecules was closely related to the number of hydrogen bonds between cellulose and water and the diffusion degree of water molecules also affected the size of the cell to a certain extent.

4. The mechanical properties first increased and then weakened with the increase of water molecular mass fraction and appeared negative when the water molecular mass fraction reached $7 \%$. In the process of heat treatment of bamboo, when the mass fraction of water vapor reached $7 \%$, the structure of bamboo was seriously damaged. Based on this finding, it is probably the best for the mechanical properties of bamboo when the mass fraction of water vapor is about $3 \%$.

\section{ACKNOWLEDGMENTS}

The authors are grateful for the support by The Fundamental Research Funds for the Central Universities (41419020) and the Science Fund Project for Studying Abroad in Heilongjiang Province (LC201407).

\section{REFERENCES CITED}

Anderson, H. C. (1980). "Molecular dynamics simulations at constant pressure and/or temperature," The Journal of Chemical Physics 72(4), 2384-2393. DOI:

10.1063/1.439486

Cao, Y., Lu, J., Huang, R., and Jiang, J. (2012). "Increased dimensional stability of Chinese fir through steam-heat treatment," European Journal of Wood and Wood Products 70(4), 441-444. DOI: 10.1007/s00107-011-0570-y

Chen, W., Lickfield, G. C., and Yang, C. Q. (2004). "Molecular modeling of cellulose in amorphous state. Part I: Model building and plastic deformation study," Polymer 45(3), 1063-1071. DOI: 10.1016/j.polymer.2003.11.020

Chen, W., Lickfield, G. C., and Yang, C. Q. (2004). "Molecular modeling of cellulose in amorphous state. Part II: Effects of rigid and flexible crosslinks on cellulose," Polymer 45(21), 7357-7365. DOI: 10.1016/j.polymer.2004.08.023

Ewald, P. P. (1921). "Evaluation of optical and electrostatic lattice potentials," Annals of Physics 64, 253-287.

Heiner, A. P., Kuutti, L., and Teleman, O. (1998). "Comparison of the interface between water and four surfaces of native crystalline cellulose by molecular dynamics 
simulations," Carbohydrate Research 306(1-2), 205-220. DOI: 10.1016/S00086215(97)10053-2

Hinterstoisser, B., Åkerholm, M., and Salmén, L. (2003). "Load distribution in native cellulose," Biomacromolecules 4(5), 1232-1237. DOI: 10.1021/bm030017k

Lundgaard, L. E., Hansen, W., Linhjell, D., and Painter, T. J. (2004). "Aging of oilimpregnated paper in power transformers," IEEE Transactions on Power Delivery 19(1), 230-239. DOI: 10.1109/TPWRD.2003.820175

Li, X., Sun, C., Zhou, B., and He, Y. (2015). "Determination of hemicellulose, cellulose and lignin in moso bamboo by near infrared spectroscopy," Scientific Reports 5, Article Number 17210. DOI: 10.1038/srep17210

LeNeveu, D. M., Rand, R. P., and Parsegian, V. A. (1976). "Measurement of forces between lecithin bilayers," Nature 259(5544), 601-603. DOI: 10.1038/259601a0

Lee, S. H., and Rossky, P. J. (1994). "A comparison of the structure and dynamics of liquid water at hydrophobic and hydrophilic surfaces-A molecular dynamics simulation study," The Journal of Chemical Physics 100(4), Article Number 3334. DOI: $10.1063 / 1.466425$

Mazeau, K., and Heux, L. (2003). "Molecular dynamics simulations of bulk native crystalline and amorphous structures of cellulose," The Journal of Physical Chemistry B 107(10), 2394-2403. DOI: 10.1021/jp0219395

Miyamoto, H., Schnupf, U., Crowley, M. F., and Brady, J. W. (2016). "Comparison of the simulations of cellulosic crystals with three carbohydrate force fields," Carbohydrate Research 422, 17-23. DOI: 10.1016/j.carres.2016.01.001

Onyon, P. (1972). "Polymer handbook," Nature 238, 56. DOI: 10.1038/238056a0

Sun, Y., Chen, L., Cui, L., Zhang, Y., and Du, X. (2018). "Molecular dynamics simulation of cross-linked epoxy resin and its interaction energy with graphene under two typical force fields," Computational Materials Science 143, 240-247. DOI: 10.1016/j.commatsci.2017.11.007

Theodorou, D. N., and Suter, U. W. (1985). "Detailed molecular structure of a vinyl polymer glass," Macromolecules 18(7), 1467-1478. DOI: 10.1021/ma00149a018

Wang, W., Wang, Y., and Li, X. (2018). "Molecular dynamics study on mechanical properties of cellulose with air/nitrogen diffusion behavior," BioResources 13(4), 7900-7910. DOI: 10.15376/biores.13.4.7900-7910

Zhang, C., Xue, X., Cao, Y., Zhou, Y., Li, H., Zhou, J., and Gao, T. (2013). "Intermolecular friction symbol derived from crystal information," CrystEngComm 15(34), 6837-6844. DOI: 10.1039/c3ce40817e

Article submitted: November 21, 2019; Peer review completed: May 224, 2020; Revised version received and accepted: July 7, 2020; Published: July 14, 2020.

DOI: 10.15376/biores.15.3.6766-6780 